\title{
The fate of S-bearing species after ion irradiation of interstellar icy grain mantles
}

\author{
M. Garozzo ${ }^{1}$, D. Fulvio ${ }^{1,2}$, Z. Kanuchova ${ }^{1,3}$, M. E. Palumbo ${ }^{1}$, and G. Strazzulla ${ }^{1}$ \\ 1 INAF - Osservatorio Astrofisico di Catania, via S. Sofia 78, 95123 Catania, Italy \\ e-mail: [mga;mepalumbo]@oact.inaf.it \\ 2 Dipartimento di Fisica e Astronomia, Università di Catania, Italy \\ 3 Astronomical Institute of Slovak Academy of Science, 059 60, T. Lomnica, Slovakia
}

Received 31 July 2009 / Accepted 8 October 2009

\begin{abstract}
Context. Chemical models predict the presence of S-bearing molecules such as hydrogen sulfide $\left(\mathrm{H}_{2} \mathrm{~S}\right)$ in interstellar icy grain mantles in dense molecular clouds. Up to now only two S-bearing molecules, namely sulfur dioxide $\left(\mathrm{SO}_{2}\right)$ and carbonyl sulfide (OCS), have been detected in the solid phase towards young stellar objects (YSOs), while upper limits for solid $\mathrm{H}_{2} \mathrm{~S}$ have been reported towards the same lines of sight. The estimated abundance of S-bearing molecules in icy grain mantles is not able to account for the cosmic S abundance.

Aims. In this paper we studied the effects of ion irradiation on different icy targets formed by carbon monoxide $(\mathrm{CO})$ and $\mathrm{SO}_{2}$ or $\mathrm{H}_{2} \mathrm{~S}$ as mixtures and, for the first time, as layers.

Methods. We carried out several irradiation experiments on ices containing $\mathrm{SO}_{2}$ or $\mathrm{H}_{2} \mathrm{~S}$ mixed or layered with CO. The samples were irradiated with $200 \mathrm{keV}$ protons in a high-vacuum chamber $\left(P<10^{-7} \mathrm{mbar}\right)$ at a temperature of $16-20 \mathrm{~K}$. IR spectra of the samples were recorded after various steps of irradiation and after warm-up.

Results. We have found that the column density of $\mathrm{H}_{2} \mathrm{~S}$ and $\mathrm{SO}_{2}$, as well as $\mathrm{CO}$, decreases after irradiation, and the formation of new molecular species is observed. In the case of CO:SO 2 samples, OCS, sulfur trioxide $\left(\mathrm{SO}_{3}\right)$, ozone $\left(\mathrm{O}_{3}\right)$, and carbon dioxide $\left(\mathrm{CO}_{2}\right)$ are the most abundant species formed. In the case of CO: $\mathrm{H}_{2} \mathrm{~S}$ samples the most abundant species formed are OCS, $\mathrm{SO}_{2}$, carbon disulfide $\left(\mathrm{CS}_{2}\right)$, hydrogen persulfide $\left(\mathrm{H}_{2} \mathrm{~S}_{2}\right)$, and $\mathrm{CO}_{2}$. The profile of the OCS band formed after irradiation of the $\mathrm{CO}: \mathrm{H}_{2} \mathrm{~S}$ mixture compares well with the profile of the OCS band detected towards the high mass YSO W33A.

Conclusions. Our results show that on a time scale comparable to the molecular cloud lifetime, the column density of $\mathrm{H}_{2} \mathrm{~S}$ is strongly reduced and we suggest that this could explain the failure of its detection in the solid phase in the lines of sight of YSOs. We suggest that the solid OCS and $\mathrm{SO}_{2}$ detected in dense molecular clouds are formed after ion irradiation of icy grain mantles.
\end{abstract}

Key words. astrochemistry - molecular processes - methods: laboratory - techniques: spectroscopic, ISM: molecules ISM: abundances

\section{Introduction}

Observations of various astrophysical environments show that ices are very common in the Universe. They can be found in the Solar System (on the surface of planets, satellites, comets and other minor objects), as well as in the interstellar medium, inside dense molecular clouds where, due to the low temperature $(\sim 10 \mathrm{~K})$ and high density $\left(\geq 10^{4} \mathrm{H} \mathrm{cm}^{-3}\right)$, gas species freeze out on dust grains forming icy mantles. When the ices are exposed to particle fluxes (cosmic ions, solar wind, etc.), they are modified chemically and structurally. Incoming ions release their energy into the target and destroy molecular bonds producing fragments that, by recombination, form new molecules, also different from the original ones and, in general, also with a new lattice structure. This explains the change of spectral behavior of irradiated surfaces (Brunetto et al. 2006) as well as the chemical composition of cometary (e.g. Strazzulla et al. 1991) or interstellar (e.g. Palumbo et al. 2000) ices. Thus, the laboratory simulations of ion irradiation on different materials (ices) is key in understanding their evolution within the Solar System and in the interstellar medium. In this paper, we have focused on the study of the alterations induced by cosmic ion bombardment of S-bearing molecules in the icy mantles of dust grains in dense molecular clouds. Until now, only two S-bearing molecules - sulfur dioxide $\left(\mathrm{SO}_{2}\right)$ and carbonyl sulfide (OCS) - have been detected in icy grain mantles (e.g. Boogert et al. 1996, 1997; Zasowski et al. 2009; Palumbo et al. 1995, 1997), while the presence of hydrogen sulfide $\left(\mathrm{H}_{2} \mathrm{~S}\right)$ has been suggested (e.g. Geballe et al. 1985) although it has never been firmly identified, probably because its main band near $3.92 \mu \mathrm{m}$ overlaps with a feature of methanol $\left(\mathrm{CH}_{3} \mathrm{OH}\right)$. The gaseous OCS molecules observed in hot cores are thought to have originated on dust grains (Hatchell et al. 1998). The parent molecules of OCS are not yet known. Ferrante et al. (2008) recently suggested that OCS is a product of ion irradiation of a mixture of $\mathrm{CO}$ and $\mathrm{H}_{2} \mathrm{~S}$ ice. For our study, we carried out several irradiation experiments on ices containing $\mathrm{SO}_{2}$ and $\mathrm{H}_{2} \mathrm{~S}$ pure or mixed with carbon monoxide (CO). Although models of interstellar ices propose that their compounds are mixed together, recent observations suggest that interstellar ices are best represented by a layered ice model rather than mixed ice (Fraser et al. 2004; Palumbo 2006). For this reason, we have performed experiments with different kinds of icy targets. Some targets were prepared by a co-deposition of a mixture of two gas compounds at low temperature $\left(\mathrm{CO}\right.$ and $\mathrm{SO}_{2}$ or $\left.\mathrm{H}_{2} \mathrm{~S}\right)$ and others by a deposition of an $\mathrm{SO}_{2}$ or $\mathrm{H}_{2} \mathrm{~S}$ layer subsequently covered with a $\mathrm{CO}$ layer. The substrate for the experiments was a 
Table 1. Details of experiments performed and discussed in this work.

\begin{tabular}{lcccc}
\hline \hline Sample & Substrate & $T(\mathrm{~K})$ & Ion & $E(\mathrm{keV})$ \\
\hline $\mathrm{CO}: \mathrm{SO}_{2}=5: 1$ mixture & $\mathrm{Si}$ & 16 & $\mathrm{H}^{+}$ & 200 \\
$\mathrm{CO} / \mathrm{SO}_{2}$ layer & $\mathrm{Si}$ & 16 & $\mathrm{H}^{+}$ & 200 \\
$\mathrm{CO}: \mathrm{H}_{2} \mathrm{~S}=10: 1$ mixture & $\mathrm{Si}$ & 20 & $\mathrm{H}^{+}$ & 200 \\
$\mathrm{CO}: \mathrm{H}_{2} \mathrm{~S}=1: 10$ mixture & $\mathrm{KBr}$ & 20 & $\mathrm{H}^{+}$ & 200 \\
$\mathrm{CO} / \mathrm{H}_{2} \mathrm{~S}$ layer & $\mathrm{KBr}$ & 20 & $\mathrm{H}^{+}$ & 200 \\
\hline
\end{tabular}

silicon wafer or a $\mathrm{KBr}$ pellet. To our knowledge, the layered ones are the first experiments ever performed to study the chemistry induced at the interface between two icy species, while irradiation experiments with different gases and gas mixtures deposited on a refractory substrate at low temperature are more common (Mennella et al. 2004; Gomis \& Strazzulla 2005, 2008; Moore et al. 2007a; Loeffler et al. 2006). Details of all the experiments performed and discussed in this work are listed in Table 1. The thickness of all the targets was smaller than the penetration depth of the used ions $\left(200 \mathrm{keV} \mathrm{H}^{+}\right)$; so, after the energy release into the material, the passing ions were implanted in the (silicon or $\mathrm{KBr}$ ) substrate on which ices had been deposited.

\section{Experimental apparatus}

The experiments were performed in a high-vacuum chamber $\left(P<10^{-7}\right.$ mbar) facing, through $\mathrm{KBr}$ windows, a FTIR spectrophotometer (Bruker Vertex 70) working in the spectral range $8000-400 \mathrm{~cm}^{-1}(1.25-25 \mu \mathrm{m})$ with a resolution of $1 \mathrm{~cm}^{-1}$. Preprepared gases (or mixtures) are admitted into the chamber by a needle valve and accreted in the form of ices on a silicon or $\mathrm{KBr}$ substrate placed in thermal contact with the tail section of a closed-cycle helium cryostat, whose temperature can vary from 10 to $300 \mathrm{~K}$. During the deposition, the thickness of the growing ice film is monitored by a He-Ne laser interference fringe system (Baratta \& Palumbo 1998; Fulvio et al. 2009). Ions are obtained from a $200 \mathrm{kV}$ ion implanter (Danfysik 1080-200) which is interfaced to the vacuum chamber. The ion beam current densities are maintained below $1 \mu \mathrm{A} \mathrm{cm} \mathrm{cm}^{-2}$ to avoid macroscopic heating of the target. The substrate plane forms an angle of $45^{\circ}$ with the IR beam of the spectrometer and the ion beam (the two being mutually perpendicular) so transmittance spectra can be easily obtained in situ, without tilting the sample. Each time, two IR spectra were recorded, selecting the electric vector parallel ( $\mathrm{P}$ polarization) and perpendicular ( $\mathrm{S}$ polarization) to the plane of incidence. The polarization is selected using a rotatable polarizer placed in the path of the infrared beam in front of the detector. It has been shown (Baratta et al. 2000; Palumbo et al. 2006) that when the band profiles recorded in $P$ and $S$ polarization are similar, the transitions are weak and the features seen in the transmission spectra directly reflect the variation of the absorption coefficient of the solid sample. This circumstance was observed for the profile of all the new bands present in the spectra after ion irradiation. For the study of these bands, $\mathrm{P}$ spectra were considered since the signal to noise ratio is higher for this polarization. On the other hand, the profile of the $\mathrm{CO}$ and $\mathrm{SO}_{2}$ bands in the layered samples are different in $\mathrm{P}$ and $\mathrm{S}$ spectra. In $\mathrm{P}$ spectra LO-TO splitting is observed (Baratta \& Palumbo 1998; Palumbo et al. 2006). All spectra shown in the following have been taken in $\mathrm{P}$ polarization.

After irradiation, targets were warmed at a rate of a few degrees/minute up to sublimation of the sample and some spectra were taken at chosen temperatures in the range 20$150 \mathrm{~K}$. For more details on the experimental setup the reader is
Table 2. Thickness and density of the samples, stopping powers and the investigated dose ranges.

\begin{tabular}{lcccc}
\hline \hline Sample & $\begin{array}{c}\text { Thickness } \\
(\mu \mathrm{m})\end{array}$ & $\begin{array}{c}\varrho \\
\left(\mathrm{g} \mathrm{cm}^{-3}\right)\end{array}$ & $\begin{array}{c}\text { Stopping }^{a} \\
\left(\mathrm{eV}^{-1}\right)\end{array}$ & $\begin{array}{c}\text { Dose range } \\
(\mathrm{eV} / 16 \mathrm{u})\end{array}$ \\
\hline $\mathrm{CO}: \mathrm{SO}_{2}=5: 1$ & 0.776 & $0.99^{b}$ & 7.5 & $1.3-80.7$ \\
$\mathrm{CO} / \mathrm{SO}_{2}$ layer & $0.480 / 0.318$ & $0.8^{c} / 1.93^{d}$ & $6.2 / 14.2$ & $1.3-39.8$ \\
$\mathrm{CO}: \mathrm{H}_{2} \mathrm{~S}=10: 1$ & 0.662 & $0.83^{b}$ & 6.6 & $1.3-42.2$ \\
$\mathrm{CO}: \mathrm{H}_{2} \mathrm{~S}=1: 10$ & 0.356 & $1.26^{b}$ & 10.0 & $1.3-42.1$ \\
$\mathrm{CO} / \mathrm{H}_{2} \mathrm{~S}$ layer & $0.325 / 0.148$ & $0.8^{c} / 1.31^{b}$ & $6.1 / 10.8$ & $1.3-42.2$ \\
\hline
\end{tabular}

Notes. ${ }^{(a)}$ Stopping power of $200 \mathrm{keV}$ protons calculated by the SRIM software (Ziegler et al. 1996) ; ${ }^{(b)}$ this work ; ${ }^{(c)}$ Loeffler et al. (2005) ; (d) Rudolph (1977) .

Table 3. List of integrated band strength $(A)$ values used.

\begin{tabular}{lccc}
\hline \hline Molecule & $\begin{array}{c}\text { Band } \\
\left(\mathrm{cm}^{-1}\right)\end{array}$ & $\begin{array}{c}A \\
\left(\mathrm{~cm} \mathrm{molecule}{ }^{-1}\right)\end{array}$ & Reference \\
\hline $\mathrm{H}_{2} \mathrm{~S}$ & 2550 & $2.9 \times 10^{-17}$ & Smith (1991) \\
$\mathrm{CO}_{2}$ & 2345 & $7.6 \times 10^{-17}$ & Yamada \& Person (1964) \\
$\mathrm{CO}$ & 2139 & $1.1 \times 10^{-17}$ & Jiang et al. (1975) \\
${ }^{13} \mathrm{CO}$ & 2092 & $1.1 \times 10^{-17}$ & Jiang et al. (1975) \\
$\mathrm{OCS}$ & 2050 & $1.5 \times 10^{-16}$ & Hudgins et al. (1993) \\
$\mathrm{CS}_{2}$ & 1520 & $9.13 \times 10^{-17}$ & Pugh \& Rao (1976) \\
$\mathrm{SO}_{2}$ & 1341 & $1.47 \times 10^{-17}$ & Garozzo et al. (2008) \\
$\mathrm{O}_{3}$ & 1040 & $1.4 \times 10^{-17}$ & Smith et al. (1985) \\
\hline
\end{tabular}

referred to Strazzulla et al. (2001). By measuring the ion fluence (ions $\mathrm{cm}^{-2}$ ), which is related to the ion current measured during the irradiation, and using the SRIM software (Ziegler et al. 1996) to calculate stopping powers, we can determinate, for each experiment, the radiation doses of the target, on a scale of $\mathrm{eV}$ per $16 \mathrm{u}$ molecule, where $\mathrm{u}$ is the unified atomic mass unit. In Table 2 details of the samples that have been used in our experiments are given. The thickness and density values have been obtained following the same procedure described in Fulvio et al. (2009).

The column density $N$ (molecules $\mathrm{cm}^{-2}$ ) of a given species was calculated using the following equation:

$N=\frac{\int \tau_{\nu} \mathrm{d} v}{A}$

where $\int \tau_{v} \mathrm{~d} v\left(\mathrm{~cm}^{-1}\right)$ is the integrated area (on an optical depth scale) calculated after subtraction of the underlying continuum and $A$ is the integrated band strength $\left(\mathrm{cm}\right.$ molecule $\left.{ }^{-1}\right)$. The $A$-values used are listed in Table 3.

In some cases, the normalized column density of molecules was calculated, i.e. the ratio $N / N i$, where $N i$ is the initial column density of the parent molecule and $N$ is the column density of the molecule at some point of the irradiation experiment.

\section{Results}

Before discussing the results obtained in this work, we summarize previous results concerning the irradiation of pure $\mathrm{CO}, \mathrm{SO}_{2}$ and $\mathrm{H}_{2} \mathrm{~S}$ ices. The results of $\mathrm{H}^{+}$irradiation of pure $\mathrm{CO}$ ice at low temperature $(10-80 \mathrm{~K})$ have been discussed by Palumbo et al. (2008). The formation of abundant carbon dioxide $\left(\mathrm{CO}_{2}\right)$ and several carbon chain oxides such as dicarbon monoxide $\left(\mathrm{C}_{2} \mathrm{O}\right)$, tricarbon monoxide $\left(\mathrm{C}_{3} \mathrm{O}\right)$ and tricarbon dioxide $\left(\mathrm{C}_{3} \mathrm{O}_{2}\right)$ is described therein. Effects of the irradiation of pure $\mathrm{SO}_{2}$ ice with protons of different energies at different temperatures (from 16 
M. Garozzo et al.: S-bearing species in the interstellar icy grain mantles

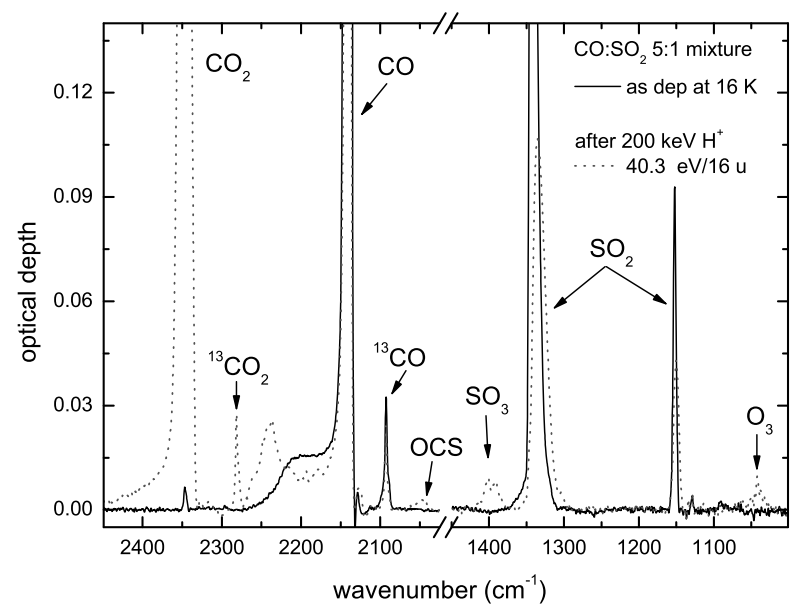

Fig. 1. IR transmittance spectra of $\mathrm{CO}: \mathrm{SO}_{2}=5: 1$ mixture as deposited at $16 \mathrm{~K}$ and after $200 \mathrm{keV} \mathrm{H}^{+}$irradiation.

Table 4. Peak position of IR bands of unirradiated $\mathrm{CO}: \mathrm{SO}_{2}$ ice as a mixture and layered sample.

\begin{tabular}{ccc}
\hline \hline \multicolumn{2}{c}{ Wavenumber $\left(\mathrm{cm}^{-1}\right)$} & Molecule \\
mixture & layered & \\
\hline 2139 & 2139 & $\mathrm{CO}$ \\
2092 & 2092 & ${ }^{13} \mathrm{CO}$ \\
1341 & 1344 & $\mathrm{SO}_{2}$ \\
1152 & 1152 & $\mathrm{SO}_{2}$ \\
\hline
\end{tabular}

to $88 \mathrm{~K}$ ) have been well studied and the formation of the sulphur trioxide $\left(\mathrm{SO}_{3}\right), \mathrm{SO}_{3}$ in a polymeric form (Moore 1984; Garozzo et al. 2008) and ozone $\left(\mathrm{O}_{3}\right.$; Garozzo et al. 2008) was observed. Results of the irradiation of pure $\mathrm{H}_{2} \mathrm{~S}$ ice at low temperature (50 and $16 \mathrm{~K}$ ) can be found in Moore et al. (2007b) and in Strazzulla et al. (2009). They agree that the only molecule detected after irradiation is hydrogen persulfide $\left(\mathrm{H}_{2} \mathrm{~S}_{2}\right)$.

\subsection{Irradiation of $\mathrm{CO}: \mathrm{SO}_{2}=5: 1$ mixture}

In Fig. 1 we plot the IR transmittance spectra of a thin film of $\mathrm{CO}: \mathrm{SO}_{2}=5: 1$ ice mixture as deposited at $16 \mathrm{~K}$ and after irradiation with $200 \mathrm{keV} \mathrm{H}^{+}$.

In the spectrum of deposited ice, the main features at 1341 and $1152 \mathrm{~cm}^{-1}$ are attributed to the $v_{3}$ asymmetric and $v_{1}$ symmetric stretching modes of the $\mathrm{SO}_{2}$ molecules, while the band at $2139 \mathrm{~cm}^{-1}$ is attributed to the $v_{1}$ stretching mode of the $\mathrm{CO}$ molecules (Table 4).

After proton irradiation, new bands appear in the spectrum, which indicate the formation of other molecules: not only abundant $\mathrm{CO}_{2}\left(2347 \mathrm{~cm}^{-1}\right)$, but also $\mathrm{SO}_{3}$ (see the doublet centred at about $\left.1400 \mathrm{~cm}^{-1}\right), \mathrm{O}_{3}\left(1041 \mathrm{~cm}^{-1}\right), \mathrm{C}_{3} \mathrm{O}_{2}\left(2193,2241 \mathrm{~cm}^{-1}\right)$ and OCS $\left(2050 \mathrm{~cm}^{-1}\right)$. A list of peak positions and the assignment of the newly formed bands is given in the Table 5 .

In Fig. 2 we report the $\mathrm{SO}_{2}$ (bottom panel) and $\mathrm{CO}$ (top panel) normalized column density as a function of the irradiation dose. The $\mathrm{SO}_{2}$ column density has been calculated using the $v_{3}$ band area $\left(\mathrm{cm}^{-1}\right)$ centred at $1341 \mathrm{~cm}^{-1}$, while the CO column density has been calculated using the band area centred at $2092 \mathrm{~cm}^{-1}$ due to ${ }^{13} \mathrm{CO}$ - the band of ${ }^{12} \mathrm{CO}$ at $2139 \mathrm{~cm}^{-1}$ being saturated. It is clear that the $\mathrm{SO}_{2}$ and $\mathrm{CO}$ column densities decrease as the dose increases, due to the fact that part of the $\mathrm{SO}_{2}$ and $\mathrm{CO}$ molecules are transformed into new species. However, the $\mathrm{SO}_{2}$ column density has a sharp drop-off followed by a much
Table 5. Peak position of the bands formed after irradiation of $\mathrm{CO}: \mathrm{SO}_{2}$ as mixture and layered sample.

\begin{tabular}{ccc}
\hline \hline \multicolumn{2}{c}{$\begin{array}{c}\text { Wavenumber }\left(\mathrm{cm}^{-1}\right) \\
\text { mixture }\end{array}$} & Molecule \\
\hline 3708 & 3708 & $\mathrm{CO}_{2}$ \\
& 2399 & $\mathrm{C}_{3} \mathrm{O}_{2}$ \\
2347 & 2346 & $\mathrm{CO}_{2}$ \\
2281 & 2281 & $13 \mathrm{CO}_{2}$ \\
& 2248 & $\mathrm{C}_{3} \mathrm{O}$ \\
2241 & 2242 & $\mathrm{C}_{3} \mathrm{O}_{2}$ \\
& 2212 & $\mathrm{C}_{5} \mathrm{O}_{2}$ \\
2193 & 2193 & $\mathrm{OCC}^{13} \mathrm{CO}$ \\
& 2180 & $\mathrm{C}_{7} \mathrm{O}_{2}$ \\
& 2123 & $\mathrm{C}_{7} \mathrm{O}_{2}$ \\
2050 & 2062 & $\mathrm{C}_{5} \mathrm{O}_{2}$ \\
& 2050 & $\mathrm{OCS}$ \\
1875 & 1989 & $\mathrm{C}_{2} \mathrm{O}$ \\
$1392-1401$ & 1875 & \\
& $1200-1402$ & $\mathrm{SO}_{3}$ \\
1041 & 1075 & $\mathrm{poly}^{2} \mathrm{SO}_{3}$ \\
659 & 1038 & $\mathrm{SO}_{3}$ \\
& 660 & $\mathrm{OO}_{3}$ \\
\hline
\end{tabular}

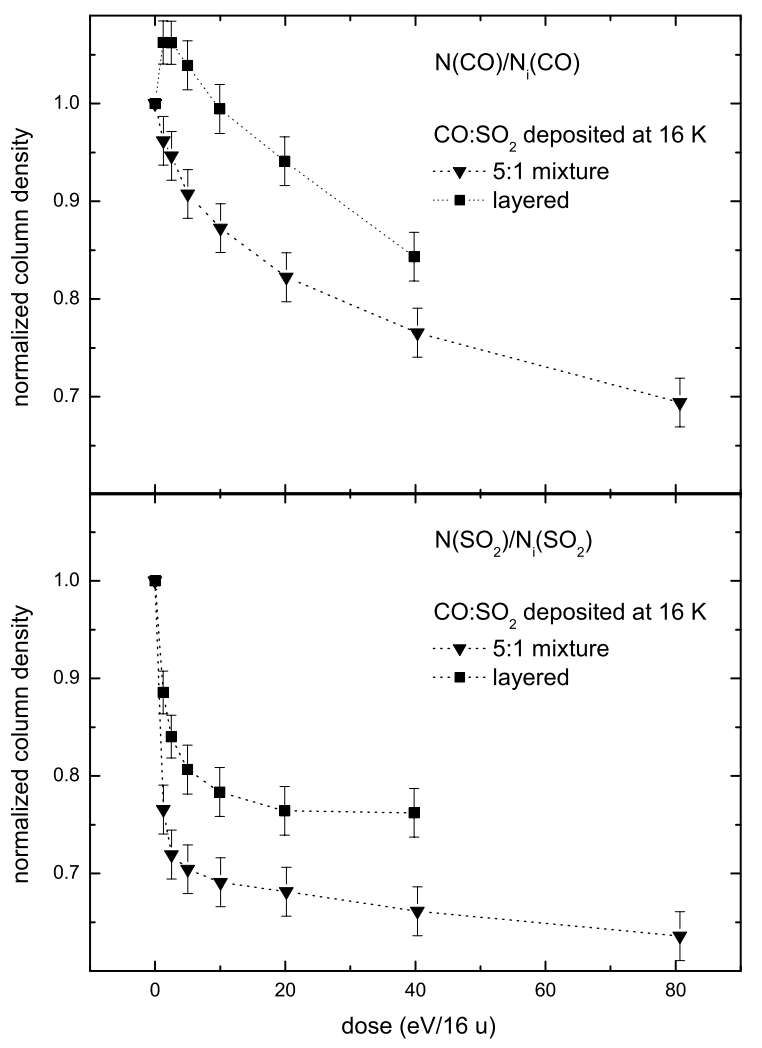

Fig. 2. Normalized column density of $\mathrm{CO}$ and $\mathrm{SO}_{2}$ as a function of dose in $\mathrm{CO}: \mathrm{SO}_{2}=5: 1$ mixture ice at $16 \mathrm{~K}$ and in $\mathrm{CO}$ deposited on $\mathrm{SO}_{2}$ at $16 \mathrm{~K}$. Points are connected for clarity.

less rapid decline. The $\mathrm{CO}$ destruction follows a more uniform behavior.

Figure 3 shows the column density of $\mathrm{CO}_{2}$, inferred from the band centred at $2347 \mathrm{~cm}^{-1}$, of $\mathrm{O}_{3}$ inferred from the band at $1041 \mathrm{~cm}^{-1}$ and of OCS (from the band at $2050 \mathrm{~cm}^{-1}$ ). To calculate these column densities we used the integrated band strength values given in Table 3. For the $\mathrm{SO}_{3}$ doublet centred at about 

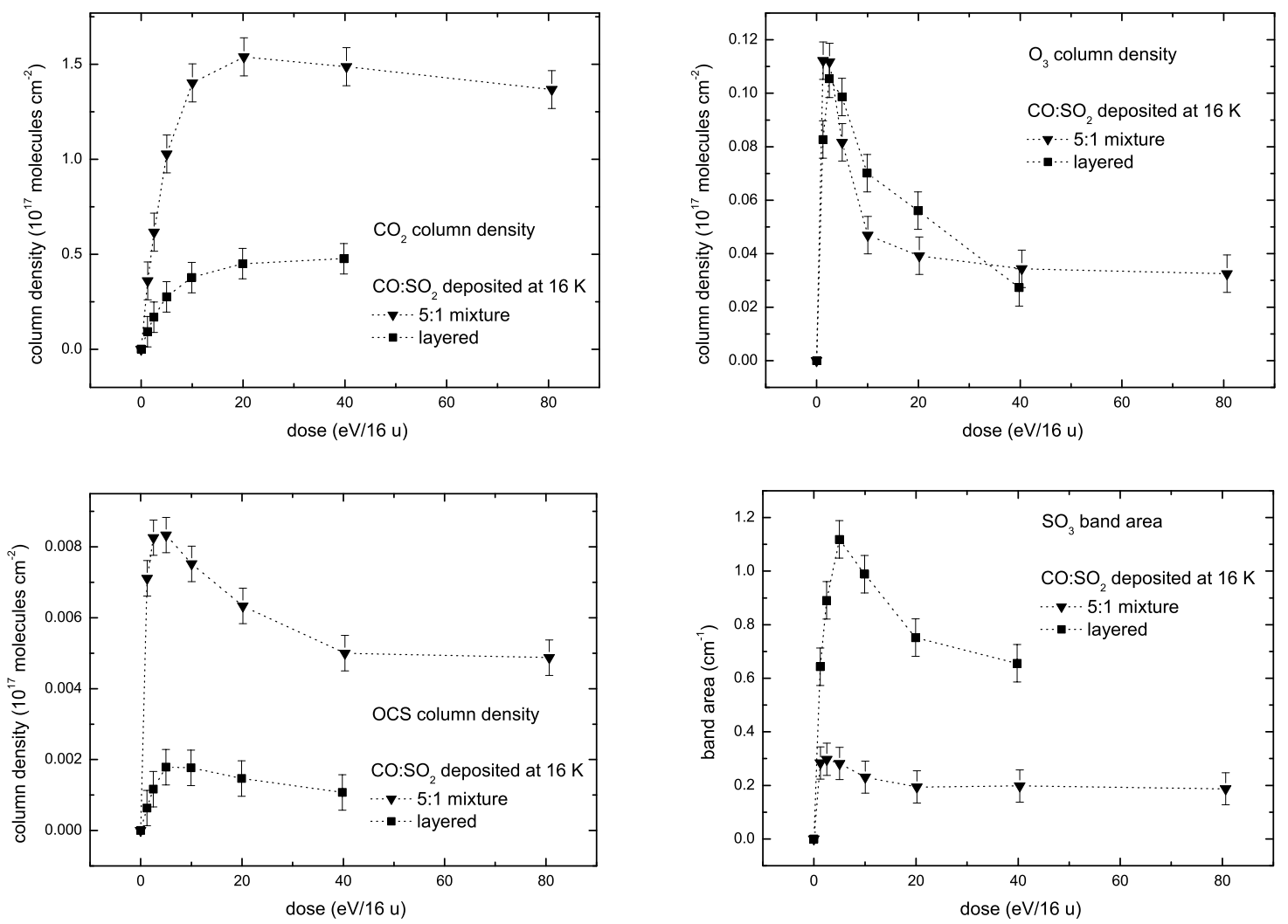

Fig. 3. The column densities of $\mathrm{CO}_{2}, \mathrm{O}_{3}$, and OCS and the band area of the $\mathrm{SO}_{3}$ doublet after $200 \mathrm{keV}$ proton irradiation of $\mathrm{CO}: \mathrm{SO}_{2}$ mixture or layered ices at $16 \mathrm{~K}$ as a function of dose. Data points are connected for clarity.

$1400 \mathrm{~cm}^{-1}$ we report the band area, because, as far as we know, the integrated band strength has not been measured.

$\mathrm{SO}_{3}, \mathrm{O}_{3}$ and $\mathrm{OCS}$ show the same trend. They increase very fast at low doses, at doses higher than $6 \mathrm{eV} / 16 \mathrm{u}$ the column densities decrease rapidly until equilibrium between destroyed and newly formed molecules is reached. This behavior is consistent with that of the parent molecule $\mathrm{SO}_{2}$ which, as said, is rapidly destroyed. The $\mathrm{CO}_{2}$ column density increases slowly and slowly decreases when the dose is higher than $20 \mathrm{eV} / 16 \mathrm{u}$.

\subsection{Irradiation of $\mathrm{CO}$ ice deposited on top of a $\mathrm{SO}_{2}$ layer}

We performed a second kind of experiment. A layer of pure $\mathrm{SO}_{2}$ was deposited onto a silicon substrate at $16 \mathrm{~K}$ and then a layer of pure $\mathrm{CO}$ was deposited onto the previous layer. This target was irradiated with $200 \mathrm{keV}$ protons. In Fig. 4 we plot the IR transmittance spectra of the $\mathrm{CO}: \mathrm{SO}_{2}$ layered ice as deposited at $16 \mathrm{~K}$ and after irradiation with $200 \mathrm{keV} \mathrm{H}^{+}$.

The main features in the spectrum of deposited ice appear at 1344,1152 and $2139 \mathrm{~cm}^{-1}$. They are attributed to the $v_{3}$ and $v_{1}$ stretching modes of the $\mathrm{SO}_{2}$ molecules and $v_{1}$ stretching mode of the $\mathrm{CO}$ molecules, respectively (Table 4 ). We notice that the profile of the $\mathrm{CO}$ band shows two peaks at $2142 \mathrm{~cm}^{-1}$ and $2139 \mathrm{~cm}^{-1}$ due to the LO and TO mode respectively (Palumbo et al. 2006). Peak positions of the newly formed bands and their assignment are listed in Table 5. The new molecules which have been formed after ion irradiation are the same as those obtained in the irradiation experiment with, separately, pure $\mathrm{SO}_{2}$ (Garozzo et al. 2008) and pure CO (Palumbo et al. 2008). In addition, OCS $\left(2050 \mathrm{~cm}^{-1}\right)$ is produced at the interface between the two layers. Also in this case we calculated the $\mathrm{SO}_{2}, \mathrm{CO}$ normalized column densities, $\mathrm{CO}_{2}, \mathrm{O}_{3}$, OCS column densities (molecules $\mathrm{cm}^{-2}$ ) and measured the $\mathrm{SO}_{3}$ band area $\left(\mathrm{cm}^{-1}\right)$ at different doses (Figs. 2 and 3). These column densities show the same trend as they have in the case of the mixture, except for $\mathrm{CO}$ that seems to increase at low doses. This is not a real growth but an effect due to the variation of the integrated absorbance of CO during irradiation. This effect was described by Loeffler et al. (2005) and it is also known for the $3 \mu \mathrm{m}$ band of water ice and has been studied by Leto \& Baratta (2003).

\subsection{Irradiation of $\mathrm{CO}: \mathrm{H}_{2} \mathrm{~S}$ mixtures}

Additional experiments were performed to study the irradiation of $\mathrm{CO}$ and $\mathrm{H}_{2} \mathrm{~S}$ ice mixtures. We have prepared two mixtures with different ratios of $\mathrm{CO}$ to $\mathrm{H}_{2} \mathrm{~S}: 10: 1$ (mixture 1) and 1:10 (mixture 2). IR transmittance spectra of mixtures 1 and 2 deposited at $20 \mathrm{~K}$ and after irradiation with $200 \mathrm{keV} \mathrm{H}^{+}$are plotted in Fig. 5.

Mixture 1: In the spectra of deposited ice there are four main features: those at 2608 and $2571 \mathrm{~cm}^{-1}$ are due to the $v_{3}$ and $v_{1}$ stretching modes of the $\mathrm{H}_{2} \mathrm{~S}$ molecules, those at 2139 and $2092 \mathrm{~cm}^{-1}$ are due to $v_{1}$ stretching modes of the $\mathrm{CO}$ and ${ }^{13} \mathrm{CO}$ molecules respectively (Table 6). The band of CO at $2139 \mathrm{~cm}^{-1}$ is saturated, thus to have information on the trend of $\mathrm{CO}$ during the irradiation we have used the band of ${ }^{13} \mathrm{CO}$.

The new molecules formed after ion irradiation of the mixture 1 (rich in $\mathrm{CO}$ ) are listed in Table 7. Two new species, OCS $\left(2043 \mathrm{~cm}^{-1}\right)$ and carbon disulfide $\mathrm{CS}_{2}\left(1519 \mathrm{~cm}^{-1}\right)$, containing both $\mathrm{C}$ and $\mathrm{S}$ atoms were identified. Other $\mathrm{S}$-bearing molecules detected after irradiation are $\mathrm{H}_{2} \mathrm{~S}_{2}\left(2486 \mathrm{~cm}^{-1}\right)$ and $\mathrm{SO}_{2}\left(1327 \mathrm{~cm}^{-1}, 1149 \mathrm{~cm}^{-1}\right)$. 
M. Garozzo et al.: S-bearing species in the interstellar icy grain mantles

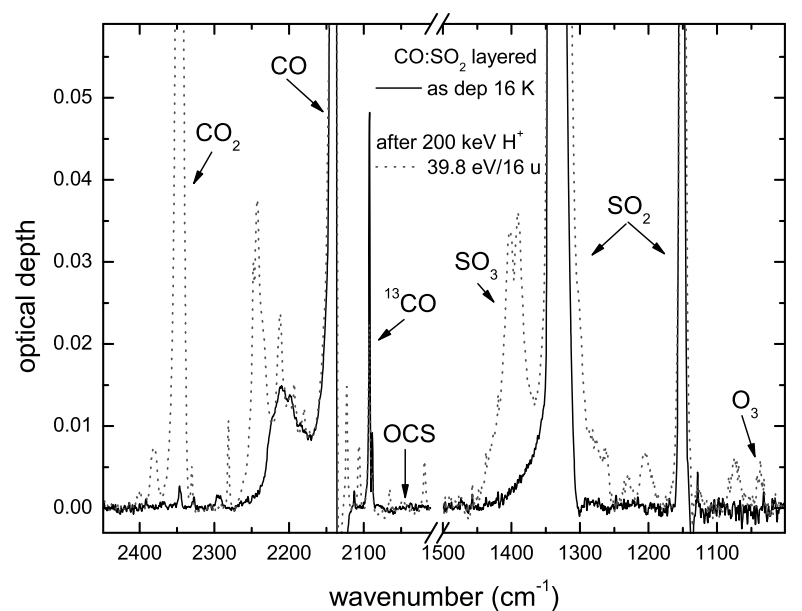

Fig. 4. IR transmittance spectra of the $\mathrm{CO}: \mathrm{SO}_{2}$ layered ice as deposited at $16 \mathrm{~K}$ and after irradiation with $200 \mathrm{keV} \mathrm{H}^{+}$.
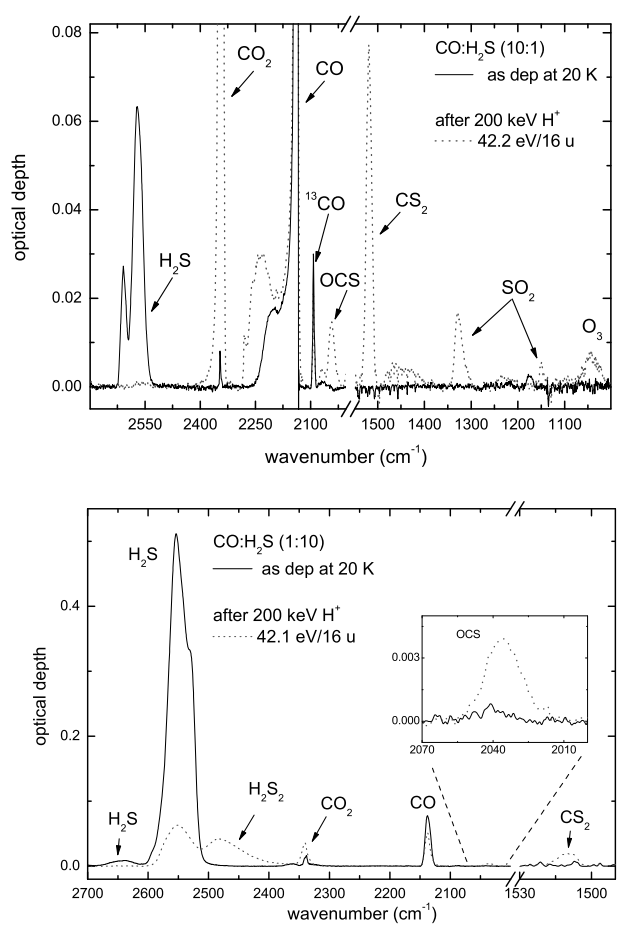

Fig. 5. IR transmittance spectra of $\mathrm{CO}: \mathrm{H}_{2} \mathrm{~S}$ 10:1 (mixture 1 - top panel) and 1:10 (mixture 2 - bottom panel) as deposited at $20 \mathrm{~K}$ and after irradiation with $200 \mathrm{keV} \mathrm{H}^{+}$.

The normalized column densities of $\mathrm{H}_{2} \mathrm{~S}$ and $\mathrm{CO}$ using the band area centred at $2571 \mathrm{~cm}^{-1}$ and $2092 \mathrm{~cm}^{-1}$ of ${ }^{13} \mathrm{CO}$ respectively are shown in Fig. 6.

Both $\mathrm{CO}$ and $\mathrm{H}_{2} \mathrm{~S}$ column densities decrease as dose increases due to the fact that these molecules are sputtered away or transformed into a new species. An extrapolated value for the sputtering yield of a surface of $\mathrm{CO}$ exposed to vacuum for $200 \mathrm{keV}$ protons is $10 \mathrm{CO} /$ proton (Brown et al. 1984), which means that about $3 \%$ of the material would have been removed at the highest fluence.
Table 6. Peak position of the IR bands of unirradiated $\mathrm{CO}: \mathrm{H}_{2} \mathrm{~S}$ ice a 10:1 mixture (mixture 1) and a 1:10 mixture (mixture 2 ) and of a layered film.

\begin{tabular}{cccc}
\hline \hline \multicolumn{3}{c}{ Wavenumber $\left(\mathrm{cm}^{-1}\right)$} & Molecule \\
mixture 1 & mixture 2 & layered & \\
\hline 2608 & 2640 & 2642 & $\mathrm{H}_{2} \mathrm{~S}$ \\
2571 & 2553 & 2546 & $\mathrm{H}_{2} \mathrm{~S}$ \\
2139 & 2138 & 2139 & $\mathrm{CO}$ \\
2092 & 2090 & 2092 & ${ }^{13} \mathrm{CO}$ \\
\hline
\end{tabular}

Table 7. Peak position of bands formed after irradiation of $\mathrm{CO}: \mathrm{H}_{2} \mathrm{~S}$ ice mixtures 10:1 (mixture 1) and 1:10 (mixture 2) and of a layered film.

\begin{tabular}{cccc}
\hline \hline \multicolumn{3}{c}{ Wavenumber $\left(\mathrm{cm}^{-1}\right)$} & Molecule \\
mixture 1 & mixture 2 & layered & \\
\hline 3707 & & 3707 & $\mathrm{CO}_{2}$ \\
2486 & 2489 & 2487 & $\mathrm{H}_{2} \mathrm{~S}_{2}$ \\
& & 2399 & $\mathrm{C}_{3} \mathrm{O}_{2}$ \\
2345 & 2338 & 2346 & $\mathrm{CO}_{2}$ \\
2281 & & 2280 & ${ }^{13} \mathrm{CO}_{2}$ \\
2258 & & & \\
2247 & & 2248 & $\mathrm{C}_{3} \mathrm{O}$ \\
2243 & & 2242 & $\mathrm{C}_{3} \mathrm{O}_{2}$ \\
2229 & & & \\
2214 & & 2212 & $\mathrm{C}_{5} \mathrm{O}_{2}$ \\
2192 & & 2192 & $\mathrm{OCC}^{13} \mathrm{CO}$ \\
2122 & & 2122 & $\mathrm{C}_{7} \mathrm{O}_{2}$ \\
2070 & & 2061 & $\mathrm{C}_{5} \mathrm{O}_{2}$ \\
2043 & 2037 & 2040 & $\mathrm{OCS}^{2}$ \\
1989 & & 1989 & $\mathrm{C}_{2} \mathrm{O}$ \\
1858 & & 1858 & $\mathrm{HCO}^{2}$ \\
1519 & 1515 & & $\mathrm{CS}_{2}$ \\
1327 & & & $\mathrm{SO}_{2}$ \\
1149 & & & $\mathrm{SO}_{2}$ \\
1044 & & & $\mathrm{O}_{3}$ \\
658 & & 659 & $\mathrm{CO}_{2}$ \\
\hline
\end{tabular}

In Fig. 6 we show the normalized column density of $\mathrm{CO}_{2}$ (from the band peaked at $\left.2345 \mathrm{~cm}^{-1}\right), \mathrm{CS}_{2}\left(1519 \mathrm{~cm}^{-1}\right)$, OCS $\left(2043 \mathrm{~cm}^{-1}\right), \mathrm{SO}_{2}\left(1327 \mathrm{~cm}^{-1}\right)$ and the band area of $\mathrm{H}_{2} \mathrm{~S}_{2}$ at $2486 \mathrm{~cm}^{-1}$ (the integrated band strength of $\mathrm{H}_{2} \mathrm{~S}_{2}$ is not known) as a function of dose.

At low doses, in agreement with the rapid destruction of the $\mathrm{H}_{2} \mathrm{~S}$ molecules, we observe the formation of new S-bearing molecules $\mathrm{H}_{2} \mathrm{~S}_{2}, \mathrm{CS}_{2}, \mathrm{SO}_{2}$ and OCS. At a dose of about $5 \mathrm{eV} / 16 \mathrm{u}$ there are not enough $\mathrm{H}_{2} \mathrm{~S}$ molecules to form new $\mathrm{H}_{2} \mathrm{~S}_{2}$, and, moreover, existing $\mathrm{H}_{2} \mathrm{~S}_{2}$ molecules are decomposed until its column density decreases to zero. OCS molecules are also slowly decomposed. The sulfur atoms, originating in $\mathrm{H}_{2} \mathrm{~S}$, are used to build up mainly $\mathrm{SO}_{2}$ and $\mathrm{CS}_{2}$ molecules. However, at the end of the experiment, the total amount of detected sulfur atoms, in the form of $\mathrm{CS}_{2}, \mathrm{SO}_{2}$ and OCS, is roughly $4 \times 10^{16}$ atoms $\mathrm{cm}^{-2}$, while at the beginning, the amount of the sulfur in the target was about $7 \times 10^{16}$ atoms $\mathrm{cm}^{-2}$ (the column density was considered). This means that a certain fraction of sulfur atoms has been sputtered and a fraction has formed a sulfur-rich residuum that is the source of sulfur atoms to build up $\mathrm{SO}_{2}, \mathrm{CS}_{2}$ and OCS after the total decomposition of $\mathrm{H}_{2} \mathrm{~S}$ (e.g. Strazzulla et al. 2009).

Mixture 2: In the spectrum of deposited ice (see Fig. 5), three main features appear: two (at 2640 and $2553 \mathrm{~cm}^{-1}$ ) are due to the $v_{3}$ and $v_{1}$ stretching modes of the $\mathrm{H}_{2} \mathrm{~S}$ molecules, and one at $2138 \mathrm{~cm}^{-1}$ is due to the $v_{1}$ stretching mode of the $\mathrm{CO}$ molecules (Table 6). The column densities of $\mathrm{CO}$ and $\mathrm{H}_{2} \mathrm{~S}$ are shown in 

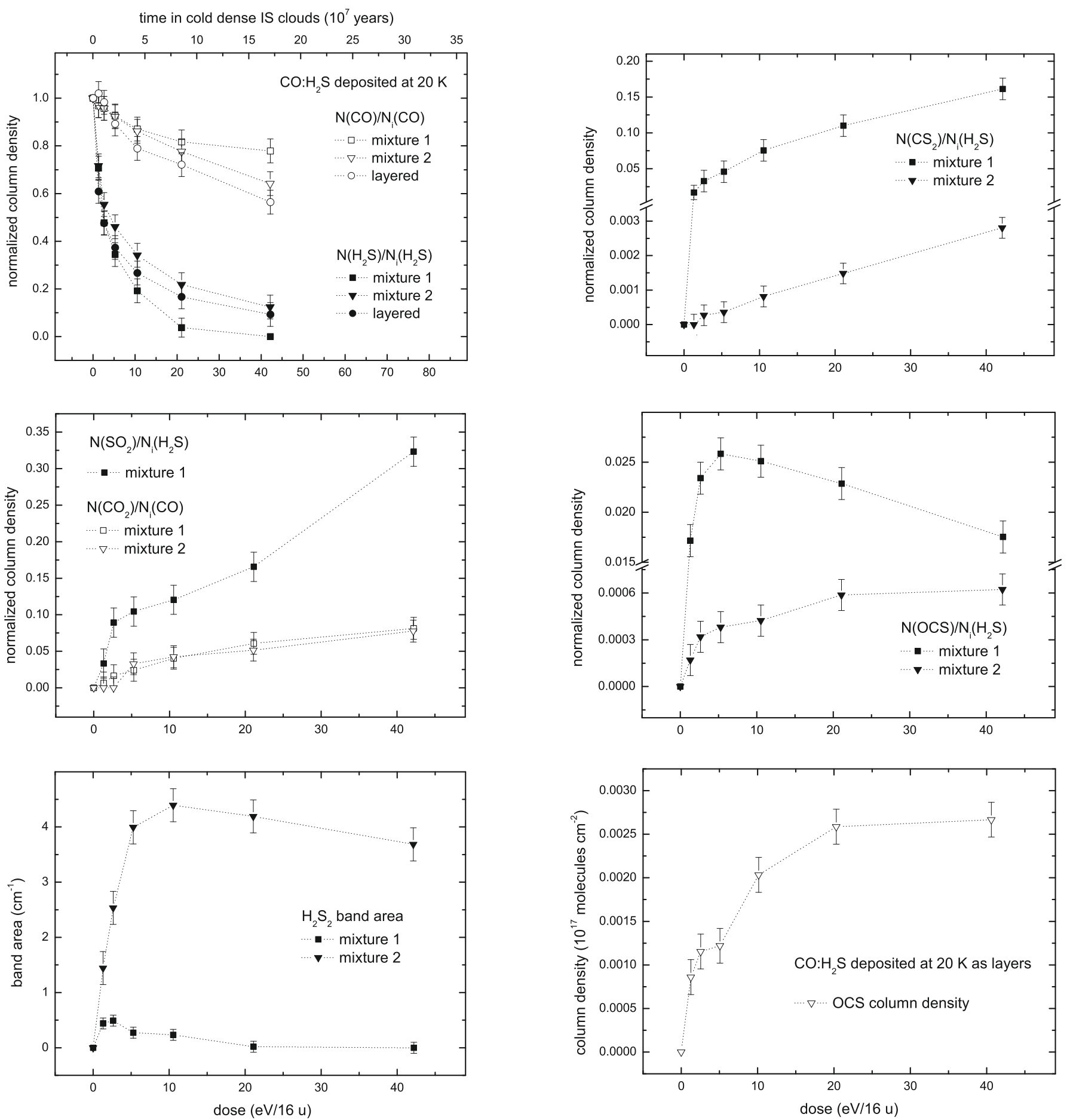

Fig. 6. Normalized column density of $\mathrm{CO}, \mathrm{H}_{2} \mathrm{~S}, \mathrm{CO}_{2}, \mathrm{CS}_{2}, \mathrm{OCS}, \mathrm{SO}_{2}$ and the band area of $\mathrm{H}_{2} \mathrm{~S}_{2}$ at $2486 \mathrm{~cm}^{-1}$ in $\mathrm{CO}: \mathrm{H}_{2} \mathrm{~S}$ mixtures 10:1 (mixture 1) and 1:10 (mixture 2) plotted as a function of dose. Column densities of CO, $\mathrm{H}_{2} \mathrm{~S}$ and OCS, in CO: $\mathrm{H}_{2} \mathrm{~S}$ layered as a function of dose are also shown. The equivalence between the doses used in laboratory and the time scale in cold dense IS clouds $(1 \mathrm{eV} / 16 \mathrm{u} \cong 4$ million years) is calculated by assuming an effective flux of monoenergetic protons $(1 \mathrm{MeV})$ of $1 \mathrm{H}^{+} \mathrm{cm}^{-2} \mathrm{~s}^{-1}$ in the IS clouds (Mennella et al. 2003). For details on calculations see Appendix A. Data points are connected for clarity.

Fig. 6 as a function of dose. As expected, the two molecular species were decomposed during irradiation, $\mathrm{CO}$ is reduced to $80 \%$ of its initial value and $\mathrm{H}_{2} \mathrm{~S}$, as in the case of mixture 1 , is almost completely destroyed. Proton irradiation causes the formation of the same molecules as in the case of irradiation of mixture 1, except for the carbon chains oxides and $\mathrm{SO}_{2}$. The normalized column densities of the molecules formed are plotted in Fig. 6 as a function of the dose. The trends of the column density of $\mathrm{CS}_{2}$ and $\mathrm{CO}_{2}$ are similar to those in the case of irradiation of mixture 1. However, the rate of decomposition and rebuilding of $\mathrm{H}_{2} \mathrm{~S}_{2}$ and OCS molecules is different with respect to the previous experiment because of the different relative amount of parent species in the two mixtures.

\subsection{Irradiation of $\mathrm{CO}$ deposited on top of $\mathrm{H}_{2} \mathrm{~S}$ ice}

An additional experiment was the irradiation of $\mathrm{CO}$ and $\mathrm{H}_{2} \mathrm{~S}$ ices in the form of layers. The target was prepared in the same 


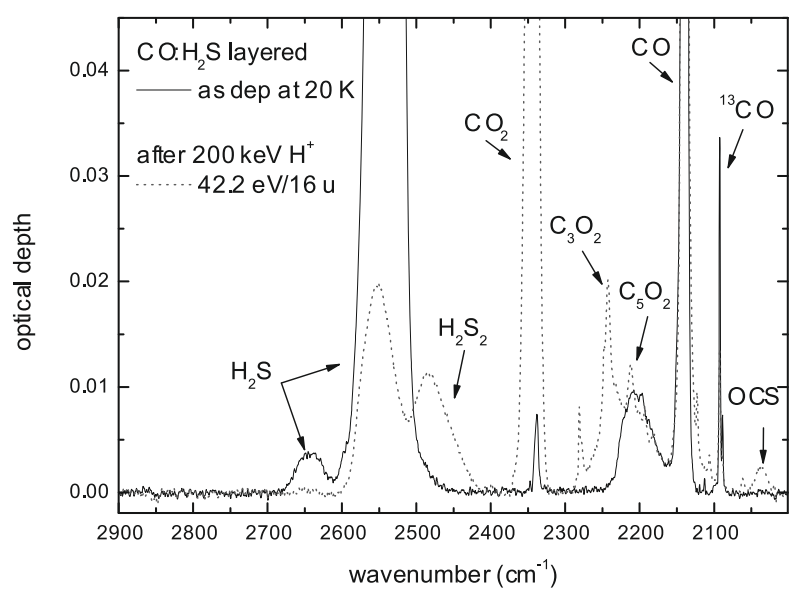

Fig. 7. IR transmittance spectra of the $\mathrm{CO}: \mathrm{H}_{2} \mathrm{~S}$ layered ice as deposited at $20 \mathrm{~K}$ and after irradiation with $200 \mathrm{keV} \mathrm{H}^{+}$.

way as the target in the experiment described in Sect. 3.2. The IR spectra of the sample before irradiation and as deposited at $20 \mathrm{~K}$ is plotted in Fig. 7. Except OCS, all the molecules formed by irradiation are the same as were formed by the irradiation of pure $\mathrm{CO}$ and pure $\mathrm{H}_{2} \mathrm{~S}$ ices. OCS molecules were formed at the interface of the two layers.

Because the column density for all the newly formed molecules follows a similar trend as in the case of pure $\mathrm{CO}$ and pure $\mathrm{H}_{2} \mathrm{~S}$ irradiation, just the OCS column density as a function of dose is presented in Fig. 6. The trend of OCS column density formed at the interface of two ices is similar to that found for the irradiated mixture rich in $\mathrm{H}_{2} \mathrm{~S}$.

\subsection{Warm-up effects}

After the end of irradiation, all icy samples were warmed up to room temperature and spectra recorded at different temperatures. During warm-up the peak position of the OCS band shifts. This shift depends on the initial mixture. As an example, in the case of the $\mathrm{CO}: \mathrm{H}_{2} \mathrm{~S}=10: 1$ mixture the peak shifts from $2043 \mathrm{~cm}^{-1}$ at $20 \mathrm{~K}$ to $2038 \mathrm{~cm}^{-1}$ at $100 \mathrm{~K}$. In this mixture the $1327 \mathrm{~cm}^{-1}$ $\mathrm{SO}_{2}$ band also shifts to lower wavenumbers after warm-up.

\section{Discussion and conclusions}

In this paper, we have studied the effects of ion irradiation of different icy targets formed by $\mathrm{CO}$ and $\mathrm{SO}_{2}$ or $\mathrm{H}_{2} \mathrm{~S}$ as mixtures and, for the first time, have separated layers. Our main results are:

1) The production of OCS is observed in all the experiments considered here using as targets $\mathrm{CO}: \mathrm{SO}_{2}$ or $\mathrm{CO}: \mathrm{H}_{2} \mathrm{~S}$ deposited as mixtures or layered. Since the thickness of the interface of two layered ices (mixing of materials) is lower than the total thickness of deposited mixtures, the total amount of produced OCS is lower in the case of ion irradiation of the layered ices. Moreover, in the cases of irradiation of CO: $\mathrm{H}_{2} \mathrm{~S}$ samples, $\mathrm{CS}_{2}$ was formed.

2) An important finding is that $\mathrm{H}_{2} \mathrm{~S}$ column density has a very rapid drop off at the beginning of irradiation, until almost all the $\mathrm{H}_{2} \mathrm{~S}$ molecules are decomposed.

3) The production of carbon chain oxides is observed only for the mixtures rich in $\mathrm{CO}$ and in the layered samples.

4) One of the products of ion irradiation of targets containing $\mathrm{SO}_{2}$ is ozone $\left(\mathrm{O}_{3}\right)$. This result confirms and extends previous findings discussed in Garozzo et al. (2008). A small amount of ozone is detected also in the $\mathrm{CO}: \mathrm{H}_{2} \mathrm{~S}=10: 1$ mixture. In this case, the formation of $\mathrm{O}_{3}$ is due to the high abundance of $\mathrm{CO}_{2}$ (Loeffler et al. 2005; Strazzulla et al. 2005) in the final stages of irradiation.

5) In the case of the $\mathrm{CO}: \mathrm{H}_{2} \mathrm{~S}$ mixtures, only in the one rich in $\mathrm{CO}$ (i.e. also rich in oxygen) are the $\mathrm{SO}_{2}$ molecules detected after irradiation. Similarly, Moore et al. (2007b) reported the formation of sulfur dioxide as a result of irradiation of ice mixtures rich in oxygen $\left(\mathrm{H}_{2} \mathrm{O}: \mathrm{H}_{2} \mathrm{~S}=8: 1\right) \cdot \mathrm{O}_{3}$ molecules also are detected only after irradiation of the $\mathrm{CO}: \mathrm{H}_{2} \mathrm{~S}=10: 1$ sample.

6) The amount of OCS produced by irradiation depends not only on the kind of sulfur-bearing species but also on the amount of $\mathrm{CO}$ involved in the process.

Ferrante et al. (2008) presented several experiments on the formation of OCS in proton irradiated ices at $10 \mathrm{~K}$. Our results are generally in agreement with those of Ferrante et al. (2008). Particularly, OCS is produced more efficiently in a mixture $\mathrm{CO}: \mathrm{H}_{2} \mathrm{~S}$, in which $\mathrm{CO}$ is more abundant with respect to $\mathrm{H}_{2} \mathrm{~S}$. As well as Ferrante et al. (2008), we have observed a shift of the OCS band peak position during heating and this shift depends on the kind of sample in which the OCS was formed. Furthermore, Ferrante et al. (2008) do not report - for their experiment on the $\mathrm{CO}: \mathrm{H}_{2} \mathrm{~S}=5: 1$ mixture - the detection of $\mathrm{O}_{3}$ and $\mathrm{SO}_{2}$ molecules. This can be due to the different mixture ratio used compared to our experiment (10:1).

So far, only two S-bearing molecules, $\mathrm{SO}_{2}$ and OCS, have been detected in the icy mantles of the dust grains of the interstellar medium (e.g. Boogert et al. 1996, 1997; Zasowski et al. 2009; Palumbo et al. 1995, 1997). In Table 8 we report the abundances measured in some Young Stellar Object (YSO) sources. Geballe et al. (1985) reported the identification of solid $\mathrm{H}_{2} \mathrm{~S}$ after detection of a band at about $3.9 \mu \mathrm{m}$ toward the high mass YSO W33A but further observations have shown that the $3.9 \mu \mathrm{m}$ band is due to methanol (Grim et al. 1991; Allamandola et al. 1992). Upper limits of $\mathrm{H}_{2} \mathrm{~S}$ column density towards other lines of sight are given in Smith (1991). The role of $\mathrm{H}_{2} \mathrm{~S}$ as a precursor of OCS and, possibly, $\mathrm{SO}_{2}$ in the icy mantles of dust grains in the interstellar medium is not well understood. $\mathrm{H}_{2} \mathrm{~S}$ in solid phase has never been identified in any YSOs, although models of envelopes of YSOs predict large quantities of $\mathrm{H}_{2} \mathrm{~S}$ on grain surfaces formed by hydrogenation of sulfur atoms (e.g. Garrod et al. 2007). The results of our experiments on targets containing $\mathrm{H}_{2} \mathrm{~S}$ show that this molecule, under the effect of ion irradiation, is reduced to $4 \%$ of its initial value after $21 \mathrm{eV} / 16 \mathrm{u}$, which corresponds to about $8 \times 10^{7}$ years in the cold and dense interstellar clouds (see Fig. 6. For the calculation of the correspondence between the irradiation dose in laboratory and the time in the dense interstellar clouds, see Appendix A). This time closely compares with the evolution time of dense clouds which ranges from 3 to $50 \times 10^{7}$ years (Greenberg 1982). This could explain the failure of $\mathrm{H}_{2} \mathrm{~S}$ solid phase detection in the interstellar medium. According to our experimental results, after the same dose (21 $\mathrm{eV} / 16 \mathrm{u})$, the missing $\mathrm{H}_{2} \mathrm{~S}$ is converted into OCS (2.3\%), $\mathrm{CS}_{2}$ $(12 \%), \mathrm{SO}_{2}(16 \%)$ and a refractory residue.

We have compared the profile of the laboratory band of OCS at different temperatures (in the interval 20 to $100 \mathrm{~K}$ ) with the band of OCS observed toward W33A. No single spectrum fits the observed OCS band perfectly, but using two components, namely that at $20 \mathrm{~K}$ (representing unheated regions) and $60 \mathrm{~K}$ (representing the dust heated by the YSO), a good fit was obtained (see Fig. 8). The use of spectra at two different 
A\&A 509, A67 (2010)

Table 8. Abundances, relative to solid $\mathrm{H}_{2} \mathrm{O}$ and total $\mathrm{H}$, of the $\mathrm{S}$-bearing species detected in the icy grain mantles.

\begin{tabular}{lcccc}
\hline \hline \multirow{2}{*}{ Source } & \multicolumn{3}{c}{ Abundances } \\
& $\mathrm{N}(\mathrm{OCS}) / \mathrm{N}\left(\mathrm{H}_{2} \mathrm{O}\right)$ & $\mathrm{N}\left(\mathrm{SO}_{2}\right) / \mathrm{N}\left(\mathrm{H}_{2} \mathrm{O}\right)$ & $\mathrm{N}(\mathrm{OCS}) / \mathrm{N}(\mathrm{H})^{a}$ & $\mathrm{~N}\left(\mathrm{SO}_{2}\right) / \mathrm{N}(\mathrm{H})^{a}$ \\
\hline Mon R2 IRS 2 & $5.5 \times 10^{-4 b}$ & & $3.2 \times 10^{-8 b}$ & \\
AFGL 989 & $1.0 \times 10^{-3 b}$ & & $2.3 \times 10^{-8 b}$ & \\
W33A & $4.0 \times 10^{-4 b}$ & $(3.1 \pm 1.6) \times 10^{-3 c}$ & $7.0 \times 10^{-8 b}$ & $(6.2 \pm 3.2) \times 10^{-7 c}$ \\
NGC 7538 IRS 1 & $<5.0 \times 10^{-4 d}$ & $(8.0 \pm 2.0) \times 10^{-3 c}$ & $<7.6 \times 10^{-9 d}$ & $(1.2 \pm 0.3) \times 10^{-7 c}$ \\
NGC 7538 IRS 9 & $5.0 \times 10^{-4 e}$ & $<5.0 \times 10^{-3 c}$ & $3.4 \times 10^{-8 e}$ & $<3.4 \times 10^{-7 c}$ \\
\hline
\end{tabular}

Notes. ${ }^{(a)} \mathrm{N}(\mathrm{H})$ column densities are taken from Tielens et al. (1991) and Boogert et al. (1997); ${ }^{(b)}$ Palumbo et al. (1997); ${ }^{(c)}$ Boogert et al. (1997); ${ }^{(d)}$ Gibb et al. (2004); ${ }^{(e)}$ Charnley et al. (2001).

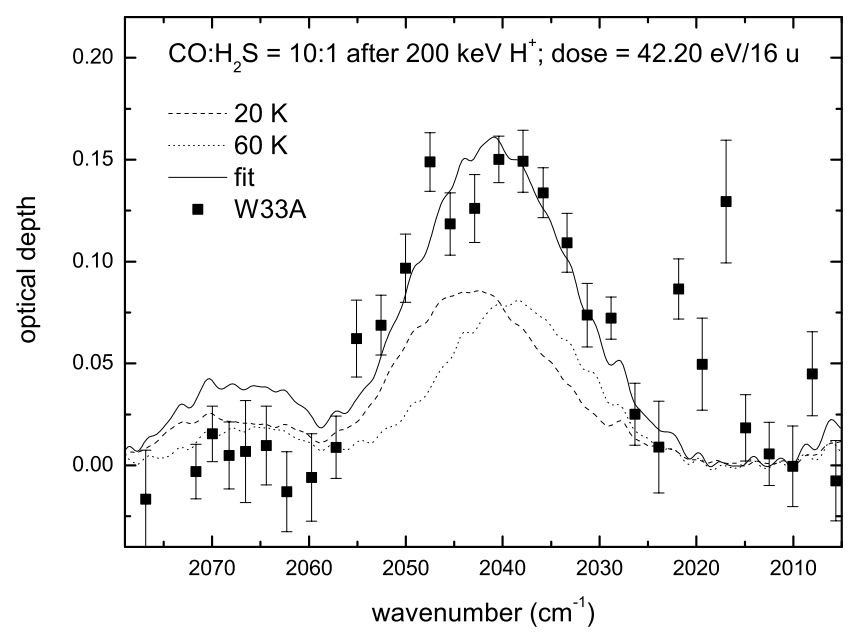

Fig. 8. Comparison between the fit obtained by the band of OCS observed in the $\mathrm{CO}: \mathrm{H}_{2} \mathrm{~S}=10: 1$ mixture at the end of irradiation at $20 \mathrm{~K}$ and warmed at $60 \mathrm{~K}$ and the band at $4.9 \mu \mathrm{m}$ in W33A observed with the IRTF (Palumbo et al. 1995).

temperatures is justified because the observed OCS band likely originates in regions of different temperature, as was demonstrated previously e.g. by Ioppolo et al. (2009) for the observed $\mathrm{CO}_{2}$ band. We also tried a quantitative comparison of the amount of OCS formed after irradiation in the mixture CO: $\mathrm{H}_{2} \mathrm{~S}=10: 1$ and the amount of OCS measured in the spectra of some YSOs. In our experiment, the ratio between the abundance of OCS and the initial abundance of $\mathrm{H}_{2} \mathrm{~S}$ is, at maximum, of about 0.025 (see Fig. 6). The fractional abundance of sulfur in the gas phase in the diffuse ISM ranges between $10^{-6}-10^{-5}$ with respect to $\mathrm{H}$, as will be discussed further in the next. If we assume that all sulfur was condensed in the ice mantles of dust grains and formed $\mathrm{H}_{2} \mathrm{~S}$ by hydrogenation, the amount of OCS that can be formed is of the order of $2.5 \times 10^{-8}-10^{-7}$, with respect to $\mathrm{H}$. This ratio is comparable to the observed values in YSOs (Table 8). As said, layered ices are presently considered a better representation of the icy mantles in interstellar dust. The profile of the OCS band obtained in our $\mathrm{CO}: \mathrm{H}_{2} \mathrm{~S}$ layered experiment is similar to that obtained from mixture 1. In Fig. 8, we show the fit with spectra from mixture 1 because they are much less noisy than those obtained from layers.

As already discussed (see point 1), in the case of CO: $\mathrm{H}_{2} \mathrm{~S}$ mixtures we observed the production of $\mathrm{CS}_{2}$. In all of the cases the relative amount of $\mathrm{CS}_{2}$ is higher (of the order of 4-6 times) with respect to OCS. Moreover, at the maximum doses reached in our experiments, we can clearly see that the produced amount of OCS reaches the saturation level while we still observe an increase of the $\mathrm{CS}_{2}$ abundance. So far, $\mathrm{CS}_{2}$ has not been observed in the solid phase in the interstellar medium and this can be due to the fact that its main band at about $6.60 \mu \mathrm{m}\left(1515 \mathrm{~cm}^{-1}\right)$ overlaps with the well-known 6.00 and $6.85 \mu \mathrm{m}$ features (e.g. Keane et al. 2001; Boogert et al. 2008). The IR spectrum of W33A in the $6.60 \mu \mathrm{m}$ region has an optical depth of about 1.3 (Schutte \& Khanna 2003), while the OCS band has an optical depth of about 0.15 (see Fig. 8). In our experiment on the $\mathrm{CO}: \mathrm{H}_{2} \mathrm{~S}$ $=10: 1$ mixture, the maximum value of the ratio between the optical depth of $\mathrm{CS}_{2}$ and OCS is 5. Thus in the spectrum of W33A a hypothetical band of $\mathrm{CS}_{2}$ should have an optical depth of about 0.75 and whose observation would be inhibited.

As reported in Table 8, the observed fractional abundance of $\mathrm{SO}_{2}$ is about an order of magnitude higher than that of OCS. Our experimental results (see Fig. 6) show that after ion irradiation, the amount of $\mathrm{SO}_{2}$ formed with respect to initial $\mathrm{H}_{2} \mathrm{~S}$ is about an order of magnitude higher than OCS.

The irradiation of targets containing $\mathrm{H}_{2} \mathrm{~S}$ led to the formation of a sulfur rich residuum (see Sect. 3) that has no IR bands, thus being not observable. We suggest that solid sulfur and $\mathrm{CS}_{2}$ produced by cosmic ion irradiation could contribute to the missing sulfur problem. Early low-resolution studies using the International Ultraviolet Explorer (IUE) gave relatively low elemental abundances of sulfur $\left(\sim 4 \times 10^{-6}\right)$, with respect to $\mathrm{H}$, in the diffuse ISM (van Steenbergen \& Shull 1988), while studies using the Goddard High Resolution Spectrograph (GHRS) on the Hubble Space Telescope revealed that most of the sulfur is in the gas phase and its abundance is $1.5 \times 10^{-5}$ with respect to $\mathrm{H}$ (Sofia et al. 1994). The most recent value of sulfur cosmic abundance with respect to $\mathrm{H}$ defined by Lodders (2003) is $1.8 \times 10^{-5}$ calculated on the basis of solar photospheric and meteoritic chondrite abundances, consistent with the observations in the diffuse interstellar medium. However, observations and gas-grain models of dense molecular clouds indicate that the gas phase sulfur abundance is about two orders of magnitude lower. For example, in the model of molecular evolution of a star-forming core, Aikawa et al. (2008) used a value of S abundance of $9.14 \times 10^{-8}$. Garrod et al. (2007) obtained $\mathrm{H}_{2} \mathrm{~S}$ gas abundance values in line with typical observed values, assuming an initial abundance of $\mathrm{S}$ of $8.0 \times 10^{-8}$, in agreement with Graedel et al. (1982). Other chemical models with low initial sulfur abundances $\left(2 \times 10^{-7}, 6 \times 10^{-9}\right)$ can be found in Millar \& Herbst (1990) and Doty et al. (2002). Thus, in molecular clouds, theoretical gas-phase chemistry models require very low elemental sulfur abundances in order to explain the observations of sulfurbearing molecules. Where is the missing sulfur? As said, we suggest that the missing sulfur is locked up in icy grain mantles as a solid residuum and/or molecules produced by cosmic ion irradiation of ices containing $\mathrm{H}_{2} \mathrm{~S}$. This latter is rapidly destroyed to produce OCS and $\mathrm{SO}_{2}$ (as observed) and other sulfur bearing molecules. 
Acknowledgements. We thank G. A. Baratta for useful discussions and F. Spinella for his help in the laboratory. This research has been supported by Italian Space Agency contract No. I/015/07/0 (Studi di Esplorazione del Sistema Solare).

\section{References}

Aikawa, Y., Wakelam, V., Garrod, R. T., et al. 2008, ApJ, 674, 984

Allamandola, L. J., Sandford, S. A., Tielens, A. G. G. M., et al. 1992, ApJ, 399, 134

Baratta, G. A., \& Palumbo, M. E. 1998, J. Opt. Soc. Am. A, 15, 3076

Baratta, G. A., Palumbo, M. E., \& Strazzulla, G. 2000, A\&A, 357, 1045

Boogert, A. C. A., Schutte, W. A., Tielens, A. G. G. M., et al. 1996, A\&A, 315, L377

Boogert, A. C. A., Schutte, W. A., Helmich, F. P., Tielens, A. G. G. M., \& Wooden, D. H. 1997, A\&A, 317, 929

Boogert, A. C. A., Pontoppidan, K. M., Knez, C., et al. 2008, ApJ, 678, 985

Brown, W. L., Augustyniak, W. M., Macartonio, K. J., et al. 1984, Nucl. Instr. Meth. Phys. Res. B, 1, 307

Brunetto, R., Barucci, M. A., Dotto, E., et al. 2006, ApJ, 644, 646

Charnley, S. B., Ehrenfreund, P., \& Kuan, Y. J. 2001, Spectrochim. Acta A, 57, 685

Doty, S. D., van Dishoeck, E. F., van der Tak, F. F. S., et al. 2002, A\&A, 389, 446

Ferrante, R. F., Moore, M. H., Spiliotis, M. M., et al. 2008, ApJ, 684, 1210

Fraser, H. J., Collings, M. P., Dever, J. W., et al. 2004, MNRAS, 353, 59

Fulvio, D., Sivaraman, B., Baratta, G. A., Palumbo, M. E., \& Mason, N. J. 2009, Spectrochim. Acta A, 72, 1007

Garozzo, M., Fulvio, D., Gomis, O., Palumbo, M. E., \& Strazzulla, G. 2008, Planet. Space Sci., 56, 1300

Garrod, R. T., Wakelam, V., \& Herbst, E. 2007, A\&A, 467, 1103

Geballe, T. R., Baas, R., Greenberg, J. M., et al. 1985, A\&A, 146, L6

Gibb, E. L., Whittet, D. C. B., Boogert, A. C. A., et al. 2004, ApJS, 151, 35

Gomis, O., \& Strazzulla, G. 2005, Icarus, 177, 570

Gomis, O., \& Strazzulla, G. 2008, Icarus, 194, 146

Graedel, T. E., Langer, W. D., \& Frerking, M. A. 1982, ApJS, 48, 321

Greenberg, M. 1982, Comets, ed. L. L. Wilkening (Tucson: The University of Arizona Press), 131

Grim, R. J. A., Baas, F., Greenberg, J. M., Geballe, T. R., \& Schutte, W. 1991, A\&A, 243, 473

Hatchell, J., Thompson, M. A., Millar, T. J., et al. 1998, A\&A, 338, 713

Hudgins, D. M., Sandford, S. A., Allamandola, L. J., et al. 1993, ApJS, 86, 713

Ioppolo, S., Palumbo, M. E., Baratta, G. A., et al. 2009, A\&A, 493, 1017

Jiang, G. J., Person, W. B., \& Brown, K. G. 1975, J. Chem. Phys., 62, 1201

Keane, J. V., Tielens, A. G. G. M., Boogert, A. C. A., Schutte, W. A., \& Whittet, D. C. B. 2001, A\&A, 376, 254

Leto, G., \& Baratta, G. A. 2003, A\&A, 397, 7

Lodders, K. 2003, ApJ, 591, 1220

Loeffler, M. J., Baratta, G. A., Palumbo, M. E., Strazzulla, G., \& Baragiola, R. A. 2005, A\&A, 435, 587

Loeffler, M. J., Raut, U., \& Baragiola, R. A. 2006, ApJ, 649, L133

Mennella, V., Baratta, G. A., Esposito, A., Ferini, G., \& Pendleton, Y. J. 2003, ApJ, 587, 727

Mennella, V., Palumbo, M. E., \& Baratta, G. A. 2004, ApJ, 615, 1073

Millar, T. J., \& Herbst, E. 1990, A\&A, 231, 466

Moore, M. H. 1984, Icarus, 59, 114

Moore, M. H., Ferrante, R. F., Hudson R. L., \& Stone, J. N. 2007a, Icarus, 190, 260

Moore, M. H., Hudson, R. L., \& Carlson, R. W. 2007b, Icarus, 189, 409
Palumbo, M. E. 2006, A\&A, 453, 903

Palumbo, M. E., Tielens, A. G. G. M., \& Tokunaga, A. T. 1995, ApJ, 449, 674

Palumbo, M. E., Geballe, T. R., \& Tielens, A. G. G. M. 1997, ApJ, 479, 839

Palumbo, M. E., Strazzulla, G., Pendleton, Y. J., et al. 2000, ApJ, 534, 801

Palumbo, M. E., Baratta, G. A., Collings, M. P., et al. 2006, Phys. Chem., 8, 279

Palumbo, M. E., Leto, P., Siringo, C., \& et al. 2008, ApJ, 685, 1033

Pugh, L. A., \& Rao, K. N. 1976, Molecular Spectroscopy: Modern research, III (New York: Academic Press)

Rudolph, R. N. 1977, Ph.D. Thesis (Boulder: University of Colorado)

Schutte, W. A., \& Khanna, R. K. 2003, A\&A, 398, 1049

Smith, M. A. H., Rinsland, C. P., Fridovich, B., et al. 1985, Molecular Spectroscopy: Modern research, III (New York: Academic Press)

Smith, R. G. 1991, MNRAS, 249, 172

Sofia, U. J., Cardelli, J. A., \& Savage, B. D. 1994, ApJ, 430, 650

Strazzulla, G., Leto, G., Baratta, G. A., et al. 1991, J. Geophys. Res., 96, 17547

Strazzulla, G., Baratta G. A., \& Palumbo M. E. 2001, Spectrochim. Acta, 57, 825

Strazzulla, G., Leto, G., Spinella, F., et al. 2005, Astrobiology, 5, 612

Strazzulla, G., Garozzo, M., \& Gomis, O. 2009, AdSpR, 43, 1442

van Steenbergen, M. E., \& Shull, J. M. 1988, ApJ, 330, 942

Tielens, A. G. G. M., Tokunaga, A. T., Geballe, T. R., et al. 1991, ApJ, 381, 181

Yamada, H., \& Person, W. B. 1964, J. Chem. Phys., 41, 2478

Zasowski, G., Kemper, F., Watson, D. M., et al. 2009, ApJ, 694, 459

Ziegler, J. F., Biersack, J. P., \& Littmark, U. 1996, The stopping and range of ions in solids. (New York: Pergamon Press, see also http: //www . srim.org)

\section{Appendix A:}

Here we define the correlation between the radiation dose used in the laboratory and the timescale in the interstellar clouds. It is important to note that chemical modification of icy target observed after ion irradiation depends, to a first approximation, on the amount of energy that ions release into the target (dose in $\mathrm{eV}$ molecule $\mathrm{e}^{-1}$ ) and not on the type of ion. Dose is defined as the product of ion flux $\left(\Phi\right.$; ions $\left.\mathrm{cm}^{-2} \mathrm{~s}^{-1}\right)$, stopping power $(S$; $\mathrm{eV} \mathrm{cm}$ molecule $\left.^{-1}\right)$ and time $(t ; \mathrm{s})$. The product of ions flux and time is the fluence (ions $\mathrm{cm}^{-2}$ ). Thus the time spent by grain mantles in the dense interstellar cloud to absorb a given dose is:

$t_{\text {ISM }}=\frac{\text { Fluence }_{\text {lab }} \times S_{\text {lab }}}{\Phi_{\text {ISM }} \times S_{\text {ISM }}}$

where the flux $\Phi$ and stopping power $S$ of the ions in the interstellar medium have to be estimated. In this case, the approximation of monoenergetic protons has been adopted. This approximation relies on the estimation of a proton flux, usually at $1 \mathrm{MeV}$, which gives rise to the $\mathrm{H}$ ionization rate produced by the cosmicion spectrum in the ISM. This flux, estimated by Mennella et al. (2003), is $\Phi(1 \mathrm{MeV})_{\mathrm{ISM}}=1 \mathrm{~cm}^{-2} \mathrm{~s}^{-1}=3.16 \times 10^{7} \mathrm{~cm}^{-2}$ years ${ }^{-1}$. The stopping power of $1 \mathrm{MeV}$ protons calculated by using the SRIM software (Ziegler et al. 1996) considering the targets used in our experiments is about 2.7 times that of the $200 \mathrm{keV}$ protons that we used in the laboratory. 